\title{
Comunicación y territorios: el caso de Tierra del Fuego
}

\author{
Communication and territories: the case of Tierra del Fuego
}

Valeria Car vcar@untdf.edu.ar

http://orcid.org/0000-0002-7969-9699

Universidad Nacional de Tierra del Fuego (Argentina)

\section{Resumen}

A partir de la década de los noventa, América Latina ha protagonizado procesos de transformaciones sociales, políticas, económicas y culturales. En este contexto, Argentina ha 
promulgado en 2009 la Ley de Servicios de Comunicación Audiovisual. Este artículo aborda las transformaciones que emergen de la aplicación de esa ley en la provincia más austral del continente americano, Tierra del Fuego. El objetivo de este estudio es describir y analizar las transformaciones y relaciones de las políticas públicas nacionales y locales en materia de Comunicación Social a partir del mapa de medios de la provincia elaborado en un trabajo previo (Ader, Car y Hermida, 2014). Los resultados contribuirán al avance de la discusión en torno a la economía política de la comunicación y la cultura a partir de las observaciones de la realidad de los medios fueguinos, el rol del Estado provincial y de la política de medios en el ámbito nacional y latinoamericano.

Palabras clave: Comunicación; medios; transformaciones; territorios; poder.

\section{Abstract}

During the last two decades, Latin America has experienced processes of social, political, economic and cultural transformation. In this context, in 2009 Argentina enacted the Law of Audiovisual Communication Services no. 26522. This article discusses the changes that emerge from the application of that law in the southernmost province of the American continent, Tierra del Fuego. The aim of this study is to describe and analyze the changes and relationships of national and local public policies on social communication, for which the provincial media map will be applied. The results will contribute to the discussion on the political economy of communication and culture, based on observations on the present situation of the fuegian media, the role of the provincial government and media policy at national and Latin American context.

Keywords: Communication; media; transformations; territories; power.

Desde el Informe McBride (1980) sabemos que la comunicación social es un derecho humano fundamental para la construcción y fortalecimiento de sociedades más humanas y más justas. Las restricciones en el acceso a la comunicación por parte de los ciudadanos en el marco de sociedades profundamente desiguales y la tendencia global a la concentración de los sistemas de medios, lleva a reflexionar acerca de la necesidad de intervenir desde la esfera pública en la regulación de las condiciones que limiten la monopolización del discurso mediático. Si bien la concentración es una característica de los mercados de capital global que se da en todo el planeta en el marco del avance de las tecnologías digitales que construyen un 
escenario complejo y convergente, las restricciones en el acceso a la comunicación se incrementan en las sociedades occidentales menos desarrolladas. Tal es el caso de la región de Latinoamérica donde se suma históricamente una evidente falta de transparencia en las relaciones entre el sector de medios y el Estado, además del acceso desigual por parte de la ciudadanía con alta concentración en pocas manos de los medios de producción y distribución de contenidos informativos y de entretenimiento (Becerra y Mastrini, 2009).

Desde una perspectiva histórica este proceso se ha caracterizado por la permanente aplicación de políticas espasmódicas que no se han sostenido más allá de los gobiernos de turno y según se trate de gestiones gubernamentales más populistas o neoliberales se han conformado condiciones de posibilidad para que la coexistencia entre gobiernos y dueños de medios se fuera consolidando en términos de estructura. Las consecuencias de esta dinámica que impide el sostenimiento de políticas públicas que atiendan a los derechos ciudadanos en materia de comunicación en el largo plazo, ha redundado en beneficios para el sector mediático cada vez más concentrado. Sin embargo, es necesario destacar que durante las últimas dos décadas se han producido algunos hechos significativos en varios países de la región que pueden sistematizarse como un conjunto de políticas más o menos afines que tienden a ubicar al ciudadano como sujeto fundamental del derecho a la comunicación por sobre los derechos comerciales. Algunos gobiernos han asumido la iniciativa, ya sea convencidos de la necesidad de regular la comunicación, ya sea para incrementar su control sobre el sistema mediático. Pero más allá de las intenciones de los gobiernos, la Conferencia Nacional de Comunicación en Brasil (2009) la Ley de Radiodifusión Comunitaria en Uruguay (2007), las Constituciones de Bolivia (2009) y Ecuador (2008), conforman diversos ejemplos de la extensión y amplitud de estos enfoques del debate.

En el contexto nacional argentino, se puede identificar algunos hitos importantes que han tensionado esa dinámica entre el Estado y el sector mediático concentrado. Así, la Ley de Servicios de Comunicación Audiovisual (2009) junto con políticas de subsidio para el sector de las industrias culturales ha sido pivote para promover las transformaciones del campo y su impacto en la sociedad. Uno de sus pilares más significativos fue la reserva del treinta y tres por ciento del espectro radioeléctrico para las organizaciones civiles sin fines de lucro, universidades, sindicatos, pueblos originarios que dicta esa Ley. Esto es un claro ejemplo destacado por la Corte Interamericana de Derechos Humanos como un caso de reivindicación concreta del derecho social a la comunicación, sin antecedentes a nivel mundial.

En este contexto de tensión y transformación, el entramado mediático de Tierra del Fuego -la provincia insular más austral de Latinoamérica y lejana de todos los grandes polos de 
producción-, se presenta como un caso a indagar para poner en evidencia algunos aspectos invisibilizados de las lógicas y dinámicas que operan territorialmente (Ader et al., 2014).

Para analizar las implicancias de estas transformaciones veremos algunos aspectos sobre cómo transversalmente estos procesos mundiales, regionales y nacionales impactan relativamente en un escenario mediático con sus características particulares de precarización en el sistema de medios local.

A continuación, expondremos el marco teórico y las discusiones en el campo de la economía y política de la comunicación que enmarcan el desarrollo del trabajo; seguidamente, situaremos desde una perspectiva histórica las implicancias de la Ley de Servicios de Comunicación Audiovisual en Argentina (2009) y posteriormente, describiremos el diseño metodológico y aplicaremos el mapa de medios de Tierra del Fuego que nos permitirá hacer visibles algunos aspectos acerca de las posibles transformaciones y tensiones en la aplicación federal de la ley.

\section{Perspectiva teórica}

El campo de la comunicación social latinoamericana ha concitado variadas y reconocidas discusiones cuyos andamiajes teóricos se inscriben y operan más desde la lógica de las intersecciones disciplinares (Canclini, 1990; Martin-Barbero, 1997; Ford, 1997; Ortiz, 2004; entre otros). La bibliografía sobre el tema no constituye un conjunto homogéneo, ya que los autores no se agrupan en una determinada forma preestablecida de prácticas intelectuales, pero sí ofrecen una perspectiva coincidente acerca de sus lugares de enunciación teórica donde los procesos de comunicación en las sociedades latinoamericanas como objetos de estudio son concebidos y analizados desde el reconocimiento de condiciones asimétricas de poder como uno de los aspectos fundamentales de su constitución.

Uno de los principales debates del campo atañe a la globalización y la cultura. Estas palabras revisten procesos complejos, problemáticos y multidimensionales donde es pausible encontrar al menos dos perspectivas contradictorias y complementarios que aportan a la comprensión de estos fenómenos. Una es la tendencia a la integración mundial que crea vínculos en procesos trasnacionales donde la sociedad avanza paulatinamente a la unidad cultural (Beck, 1998). La segunda tendencia es la revalorización de las culturas locales, la fragmentación de las identidades sociales, la reinvindicación de lo folclórico como lo "auténtico" cuyas bases filosóficas se cimentan en el pensamiento romántico, cuestiones que han sido lo saldadas en los aportes intelectuales del campo de los Estudios Culturales desde la perspectiva de la comunicación y cultura popular (Hoggart, 1957; Hall, 1984; Williams, 1989; entre otros). En 
Latinoamérica, encontramos algunos correlatos fuertes de esta perspectiva en la obra Mundialización y Cultura, de Renato Ortiz (1997), que nos permite centrar la atención en las múltiples correspondencias, articulaciones, tensiones y contradicciones entre lo local, lo nacional y lo global, contextualizado en el escenario latinoamericano a partir de su idea sobre la transformación fundamental del tiempo y espacio. En consonancia con este autor, nuestro trabajo se apoya en la idea del pensamiento complejo que exige mantener simultáneamente abiertos los planos de lo local y lo global en sus intersecciones con lo nacional. Así, este enfoque nos permitirá abordar el proceso comunicacional del entramado mediático en Tierra del Fuego poniendo en evidencia el proceso en el que se sostienen en las relaciones de complementariedad y oposición de los tópicos de centros y periferias, concentración de estructuras económicas y dispersiones mediáticas, entre otros aspectos.

Por otro lado, la complejidad del objeto de estudio requiere un abordaje desde los aportes de otras disciplinas. En este sentido, se reconoce la necesidad de introducir en estas perspectivas teóricas latinoamericanas un diálogo necesario y un acercamiento al campo específico de la economía política de la comunicación. El panorama de estos estudios es muy amplio y hay también, por supuesto, diferentes miradas, tradiciones y matices, pero sin duda los campos de las ciencias políticas, la economía política de la comunicación y el derecho (Sierra, 2006; Graziano, 1986; Fox y Waisbord, 2002; Sinclair, 2000; Becerra y Mastrini, 2007; 2009; 2011; Becerra, 2015; entre otros) aportan un insumo fundamental para comprender los procesos estructurales de la comunicación en la región. Para avalar esta necesidad, Sierra señala:

Como disciplina de estudio, la investigación en Políticas de Comunicación aborda su objeto material desde una mirada pluridisciplinaria entre la Ciencia Política, la Estructura de la Información, la Comunicación y el Desarrollo, el Derecho y la Economía Política de la Comunicación (2002: 27).

El campo de la comunicación también ha realizado contribuciones en términos de investigaciones empíricas. Para nuestro estudio son relevantes los avances producidos por Becerra y Mastrini $(2011,2014)$ quienes presentan importantes trabajos sobre el acceso y la concentración de medios en América Latina en los primeros años del siglo XXI. Allí se verifica que uno de los principales rasgos identitarios de las industrias mediáticas de la región son el progresivo incremento de su concentración y una falta de transparencia histórica en el vínculo entre los propietarios de los medios de comunicación y el Estado. Por otra parte señalan que en relación al acceso, existe una enorme brecha de desigualdad en la población latinoamericana comparativamente en relación con los países desarrollados. En consecuencia, 
la mayoría de estos países presenta una parte importante de la población marginada del consumo de bienes y servicios info comunicacionales por cuestiones económicas. Asimismo muestran que, en términos de la composición del acceso al interior de la región, se reconocen cuatro subconjuntos. En primer lugar, los tres países del Cono Sur (Argentina, Uruguay y Chile) que cultivan historia de mayores niveles comparativos de acceso social a los medios, a las industrias culturales y a las comunicaciones. En segundo lugar, Colombia, Venezuela y Perú que se ubican por arriba de media regional. En tercer lugar, Brasil y México que son los dos gigantes de población y, por último, Ecuador, Paraguay y Bolivia, países cuyo acceso social fue más relegado y muy por debajo de la media regional (Mastrini y Becerra 2011: 55-59).

Por la naturaleza del sector pero también por las condiciones inherentes a cualquier sistema capitalista en la actualidad, Latinoamérica en términos comparativos no es particularmente atractiva como región, debido a que no presenta un mercado ni con alto poder adquisitivo ni con población en gran escala (la población no alcanza a más de 50 millones, a excepción de Brasil y México). Estas características, que ubican a América Latina en desventaja en relación a las inversiones globales con los países centrales, la posicionan desfavorablemente en escala planetaria. Frecuentemente, en los países desarrollados los mercados son de gran tamaño (como Estado Unidos, Gran Bretaña, Alemania o Italia) o bien de altos ingresos (como Suecia, Bélgica, Dinamarca, Holanda). Por consiguiente, debemos soslayar que los países que integran la región latinoamericana se caracterizan, en términos amplios, por poseer históricamente una gran brecha de desigualdad que se proyecta en los modos de configuración de los sistemas de medios y su acceso por parte de la ciudadanía.

Desde esta perspectiva, proponemos nutrir la discusión del campo con la descripción y el análisis del caso de Tierra del Fuego.

\section{Metodología del caso: armando el rompecabezas de Tierra del Fuego}

Para poder observar y analizar la realidad de los medios fueguinos desde una perspectiva trasversal de los procesos socio-comunicacionales, se debió relevar, sistematizar y caracterizar el entramado mediático de Tierra del Fuego a partir de un relevamiento cuantitativo de tipo censal, utilizando como base registros administrativos que fueron ampliados con una indagación estructurada en un cuestionario cerrado (Ader et al., 2014). La muestra de origen fue ampliada con metodologías de exploración del espectro televisivo, radiofónico y de medios gráficos, apoyado en informantes claves lo que permitió alcanzar la totalidad de las unidades de análisis del universo de estudio. Dada la inexistencia de datos locales sistematizados, se 
puso en evidencia la necesidad de construir algunos indicadores que puedan dar cuenta de cuántos medios existen en la provincia, establecer parámetros comparativos en la región patagónica para valorar esa composición, conocer el grado de formalización como empresas de medios (sus formas de financiamiento, el nivel de formación profesional existente de los equipos de trabajo, las adecuaciones a las exigencias de la Ley de Servicios de Comunicación Audiovisual (2009) en aspectos relevantes del territorio como la producción de contenidos locales) y la necesidad de determinar en definitiva si la dinámica específica que caracteriza el sistema de medios de Tierra del Fuego se encuentra estructurado en lógicas que puedan promover condiciones de transformación para el desarrollo de una industria cultural sustentable. Estas zonas de indagación se agruparon bajo tres tópicos de análisis: cuantificación, formalización e integración. Finalmente se incorporan al corpus de análisis, registros documentales legales y periodísticos que nos permitieron incorporar aspectos centrales de los profundos cambios en la orientación de las políticas de comunicación social a partir de la gestión gubernamental que asumió en diciembre de 2015 en Argentina.

\section{La legislación mediática en Argentina: historia de un final incierto}

La historia de las políticas de comunicación en Argentina, la anacrónica promulgación de diferentes leyes y decretos, los muy gubernamentalizados mecanismos de control en ese campo, la escasa y poco sistematizada información pública marcada por relaciones poco trasparentes entre el sector de medios y los gobiernos de turno tienen su correlato en la historia social Argentina (Argumedo, 1985; Graziano, 1986; Postolsky, 2003; Mastrini y Becerra, 2006). En toda su historia, hubo cuatro leyes de radiodifusión. Solo la de 1953 tuvo trámite parlamentario. Las otras tres fueron decretadas por gobiernos militares. Estas marchas y contramarchas de las políticas de medios en el país tienen un punto de inflexión con la Ley 26522 de Servicios de Comunicación Audiovisual sancionada el 10 de octubre de 2009. Se puede apreciar que el panorama político y económico justificaba por demás la sanción de una nueva ley de radiodifusión acorde a las necesidades del conjunto de la sociedad. Sólo la capacidad de lobby de los grandes grupos empresarios pudo mantener una legislación tan restrictiva durante tantos años. Así, la principal simiente se remonta a 2004, momento en que se constituyó la Coalición por una Radiodifusión Democrática, responsable del Manifiesto 21 puntos básicos por el derecho a la comunicación, el cual reivindica una nueva legislación que garantice el pluralismo informativo y cultural y la existencia de medios públicos fuertes, al servicio de los ciudadanos y no de los gobiernos. El documento, a partir del entendimiento de la 
comunicación como un bien público, expresa la necesidad de promover contenidos diversos en los medios que garanticen la participación ciudadana y atiendan las necesidades de la población. De esta manera la ley recoge, por primera vez, el deber de respetar y promover el pluralismo político, religioso, social, cultural, lingüístico y étnico.

La Ley de Servicios de Comunicación Audiovisual se discutió en foros a lo largo y ancho del país durante tres largos años, erigiéndose desde su concepción en una verdadera federalización del proceso en el que las asociaciones sin fines de lucro, universidades, pueblos originarios, entre otros actores, tuvieron plena participación en el debate. De esta manera, podemos encontrar en la letra de la ley elementos muy destacables en materia de regulación democrática de la comunicación. El principal y casi inédito en el mundo, es la reserva del treinta y tres por ciento de todo el espacio radioeléctrico a organizaciones sin fines de lucro que sigue vigente en la actualidad. Basada en una doctrina de Derechos Humanos, la ley procuraba también generar una autoridad de aplicación más pluralista y transparente, a la vez que presentaba una política que limita la concentración en la propiedad. Paralelamente, se había vinculado al sistema de radiodifusión con el desarrollo y financiamiento de otras industrias culturales ya que parte de los gravámenes que deben abonar los radiodifusores por el uso del espectro, debe ser girado por la Autoridad Federal a la industria del cine y a la de la música. El proceso de implementación -que no se desarrollará en este apartado- duró desde 2009 hasta 2015 y motiva aún grandes debates y fuertes críticas en el marco de una discusión ideológica binaria y maniquea entre gobierno de turno y Grupo Clarín (grupo más concentrado de multimedios en Argentina). El lugar de la comunicación como derecho humano ciudadano que versa la letra de esa ley, así como su proceso federal de elaboración no tiene antecedentes en la dinámica pendular de políticas públicas cortoplacistas argentinas. Lamentablemente, según avanzan los meses de gestión del nuevo gobierno de turno (desde diciembre de 2015, en este caso de centro derecha) se han ido suprimiendo sistemáticamente los aspectos más destacados y paradigmáticos a través de Decretos de Necesidad y Urgencia 236/15; 267/15; 1340/16 entre otros, afectando sensiblemente la restricción a la concentración de propiedad de medios y re-gubernamentalizando nuevamente el ejercicio del Organismo de Control en el marco de un proceso que incrementa su incertidumbre sobre la continuidad de la reserva del 33 por ciento de espectro a instituciones sin fines de lucro y los mecanismos de financiamiento impositivo para la producción de contenidos en los medios audiovisuales.

Acerca de Tierra del Fuego: sistema de medios en transformación 
En un contexto de mundialización y globalización (Ortiz, 2004), la compresión de la configuración espacial se torna especialmente significativa debido a las profundas transformaciones sociales, económicas, tecnológicas y políticas durante los últimos treinta años. Por lo tanto, consideramos necesario caracterizar la complejidad del escenario territorial que pretendemos analizar. Tierra del Fuego es un territorio insular en el extremo sur de la Patagonia Argentina que se caracteriza por ser una provincia joven puesto que recién se constituyó como tal en 1991.

Esta dinámica de su poblamiento aportará a la comprensión de las formas específicas que adquiere en el territorio la aplicación de la Ley de Servicios de Comunicación Audiovisual en relación a tres tópicos de análisis: cuantificación, formalización e integración. En términos de cuantificación la provincia presenta un total de setenta y ocho (78) medios, de los cuales cincuenta y ocho (58) son radios FM, trece (13) medios gráficos (que incluyen diarios y revistas) y siete (7) canales de televisión. (Ver gráfico 1)

Gráfico 1. Medios de comunicación según su distribución por ciudades (Tierra del Fuego).

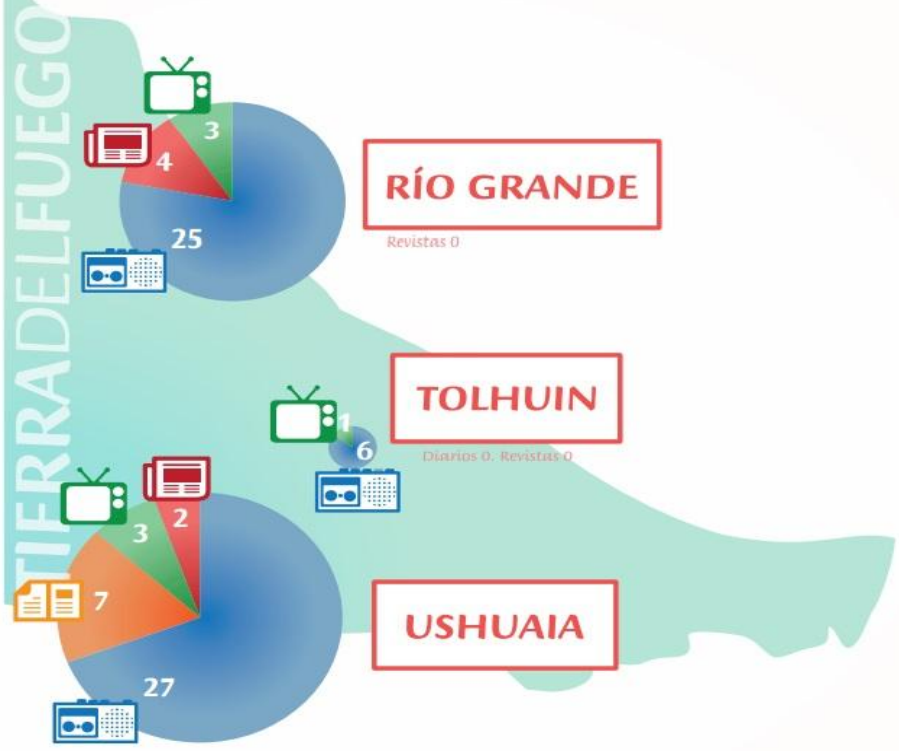

Fuente: elaboración propia. UNTDF, 2014.

La cuantificación de los medios, nos permitió confrontarla con un relevamiento realizado por la UNPA (Universidad Nacional de la Patagonia Austral, 2013) donde se evidencia que casi se duplican la cantidad de medios en Tierra del Fuego en relación a su vecina provincia de Santa Cruz. Tierra del Fuego cuenta con 5,42 medios por cada 10.000 habitantes, cuando Santa Cruz tiene 3,27 (Ader et al., 2014: 5).

Question, Vol. 1, N. ${ }^{\circ}$ 63, julio-septiembre 2019. ISSN 1669-6581 
En relación al tópico de formalización, la gran mayoría de los medios, cincuenta y tres (53) son unipersonales en su constitución jurídica con menos de tres empleados en cada caso (ver Gráfico 2). La densidad de este dato es fundamental para comprender el estado de precarización del entramado mediático local puesto que para brindar un servicio de interés público, producir en un estándar de calidad broadcasting, cumplir con la ley ( en proceso de extinción) para producir una cuota propia de contenidos, se debe contar con una cantidad mínima de personas profesionales y técnicas que cubran los diversos y centrales roles que necesitan los medios de comunicación para garantizar su funcionamiento. Se suman a esta condición que la gran mayoría de los propietarios dicen no reinvertir en su emprendimiento además de no ser en muchos casos, su principal fuente de ingreso. Como hemos visto hasta aquí, la excesiva cantidad de medios en el territorio no significa que dicho sector se encuentre en desarrollo, especialmente cuando del total de medios encuestados, sólo 28 dicen tener algún proyecto para reinvertir (Ader et al., 2014: 7). Es probable que un medio bajo la predominancia de algunos de estos tres aspectos descriptos (poco personal, baja reinversión y con otra actividad como ingreso principal) sea considerado como un medio en un estado de precariedad. 
Gráfico 2. Mapa de medios de comunicación de Tierra del Fuego.

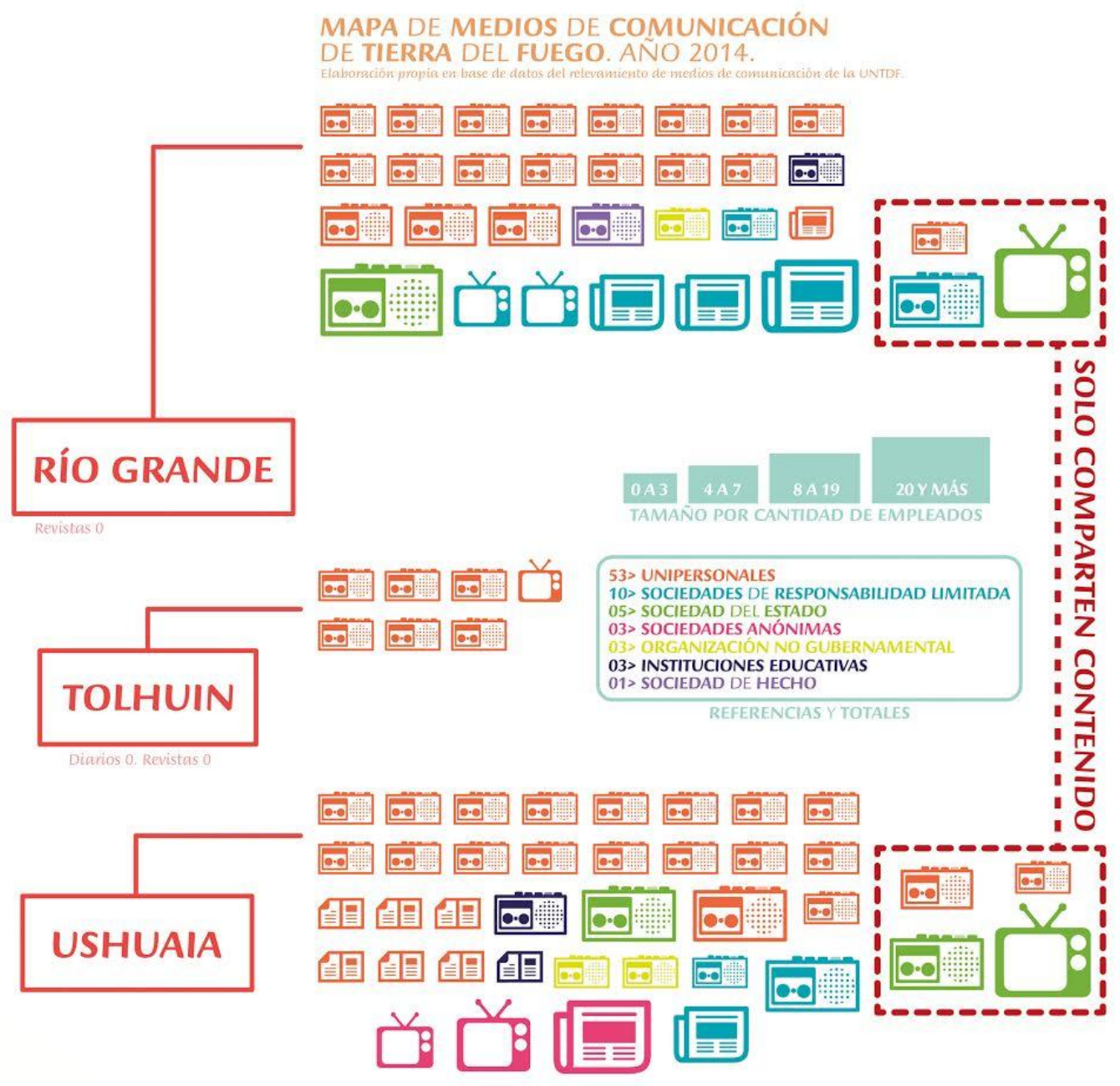

Fuente: elaboración propia, UNTDF, 2014.

Por último, desde el tópico de integración se observa que cada uno de los elementos que componen este mapa demuestran una gran dispersión mediática con una casi duplicación de medios entre ciudades, solo cuatro (4) medios comparten contenidos entre ciudades y del total de los medios, solo diecinueve (19) comparten su estructura de propiedad, cinco (5) con no más de dos (2) medios; mientras que cincuenta y cinco (55) medios cuentan con un (1) solo dueño cada uno. Se puede observar que la realidad de los medios fueguinos está atravesada por otras lógicas que distan mucho de la concentración en la estructura de propiedad sino por condiciones características de regiones periféricas. Ahora bien, una posible explicación a la 
escasa concentración de los medios fueguinos puede estar asentada en la baja densidad poblacional de la provincia y a su característica periférica de las grandes metrópolis. Es así que observamos una lógica que se presenta como subsidiaria al mismo proceso donde los medios de la provincia ejercen el rol de repetidoras de contenidos producidos en esas zonas centrales (Buenos Aires, Córdoba y Rosario). Por lo tanto la aplicación de la Ley de Servicios de Comunicación Audiovisual debe pensarse desde este contexto. En este aspecto la aplicación de la Ley interviene directamente limitando la transmisión en red de contenidos, a partir de la regulación de producción de contenidos locales.

En síntesis, como resultado de este relevamiento es plausible sostener que el entramado mediático de la provincia de Tierra del Fuego se encuentra en un estado de precarización asociado no a una lógica de desarrollo sino de subsistencia.

\section{Conclusiones}

Un aspecto central que arroja este trabajo es, sin duda, la necesidad de reconocer la enorme brecha de desigualdad social en la que se enmarca cualquier posible transformación hacia la democratización comunicacional de sociedades profundamente desiguales como las de Latinoamérica. Esta característica identitaria de la región tiene su correlato en las restricciones en el acceso a la comunicación social por parte de la ciudadanía. En el mismo sentido, las diferentes políticas y regulaciones en el campo no han cambiado sustancialmente la tendencia del sector a incrementar la concentración de los medios, incluso durante las últimas dos décadas cuando se han aplicado políticas públicas y regulaciones que pusieron en discusión, los intereses del sector de empresas de comunicación e infoentretenimiento. Sin embargo, en Tierra del Fuego se ha evidenciado que la realidad de los medios fueguinos está atravesada por otras lógicas que distan mucho de la concentración de las estructuras de propiedad de los medios. Asimismo, se ha hallado que el entramado mediático local se caracteriza por una dinámica asociada a una dispersión mediática con un gran número de medios, pero cuyo funcionamiento se encuentra estructurado sobre una lógica de subsistencia, en estado permanente de precarización.

Consideramos que este estado de precarización del entramado mediático local es otro aspecto central constitutivo y parte de la misma lógica subsidiaria de los procesos de concentración en regiones centrales ya que el bajo nivel de inversión y formalización del sector, su dinámica fragmentaria, la reducida producción de contenidos locales que llevan a la réplica de discursos de medios en posición dominante en el mercado aparecen como una opción recurrente para la 
subsistencia de los medios. Por consiguiente, es plausible afirmar que la relación histórica que constituyó el modelo de desarrollo económico y social en términos nacionales refuerza las condiciones para que prolifere un estado de dispersión mediática local, lo que lesiona el derecho de los ciudadanos fueguinos a la comunicación social.

Visibilizar estos aspectos del proceso es resituar en la agenda pública de la región, la discusión sobre el derecho humano a la comunicación desde una perspectiva que permita ampliar la mirada en términos simbólicos, pero sobre todo, territoriales ya que refuerzan estructuralmente la relación entre centro y periferia en sus mecanismos asimétricos a partir de dinámicas específicas de las industrias culturales. En este sentido, promover condiciones para una transformación del sector requiere reconocer que los entramados mediáticos lejos de las dos o tres grandes urbes del país presentan una dinámica complementaria pero muy diferente a las lógicas de concentración de esos emplazamientos urbanos. Conocer estas realidades permite jerarquizar políticas específicas asociadas a la promoción y desarrollo de la industria cultural en un sentido federal y no solo simbólico o discursivo. Por tanto, pareciera que es de vital importancia una ley que contemple estas realidades y se proyecte en el largo plazo a partir de políticas públicas sostenidas y más allá de los gobiernos de turno: una ley que no solo limite la concentración, sino que promueva la producción de contenidos locales de calidad en el marco de políticas de animación para el sector.

En síntesis, los avances en materia de comunicación social que promovió la ley debieran ser leídos como uno de los hitos más importantes para reflexionar acerca acciones que el Estado ha promovido como política de comunicación en materia de ampliación de derechos ciudadanos. Sin embargo, no solo están los interrogantes sobre la causa de las recurrencias pendulares en nuestras políticas en las que las omisiones y retrocesos -como sucede en la actualidad con esta misma ley- que son parte constitutiva y dominante en nuestra historia, sino que aparece como fundamental el re-conocimiento de los territorios en el marco de políticas de comunicación efectivamente federales.

\section{Bibliografía}

Ader, N.; Car, V. y Hermida, M. (2014). Entre medio y medio hasta el Garibaldi llego. Sociedad Fueguina, 4(2). Recuperado en http://www.untdf.edu.ar/uploads/archivos/04 Sociedad Fueguina Nro 4.pdf Argumedo, A. (1985). Los laberintos de la crisis. América Latina: poder transnacional y comunicaciones. Buenos Aires: Folios/ILET. 
Becerra, M. y Mastrini, G. (2007). La concentración mediática argentina: De eso no se habla. Buenos Aires: Centro de Estudios Legales y Sociales.

Becerra, M. y Mastrini, G. (2009). Los dueños de la palabra. Buenos Aires: Prometeo.

Becerra, M. y Mastrini, G. (2011). Estructura, concentración y en los medios del Cono Sur latinoamericano. Comunicar, revista científica de educomunicación, XVIII(36). Recuperado de http://www.revistacomunicar.com/index.php?contenido=detalles\&numero=36\&articulo= $\underline{36-2011-07}$

Becerra, M. (2015). De la concentración a la convergencia. Buenos Aires: Paidós.

Beck, U. (1998). ¿Qué es la globalización? Falacias del globalismo, respuestas a la globalización. Barcelona: Paidós.

Ford, A. (1997). La marca de la bestia: identificación, desigualdades e infoentretenimiento en la sociedad contemporánea. Buenos Aires: Grupo Editoral Norma.

Fox, E. y Waisbord, S. (2002). Latin politics, global media. Austin: University of Texas Press.

García Canclini, N. (1990). Culturas híbridas: estrategias para entrar y salir de la modernidad. México: Paidós.

Graziano, M. (1986). Política o ley: debate sobre el debate. Buenos Aires: Espacios.

Hall, S. (1984). Notas sobre la desconstrucción de «lo popular». Barcelona: Crítica.

Hoggart, R. (1990). La cultura obrera en la sociedad de masas. México: Grijalbo.

Kauffer, E. (2002). Las políticas públicas: algunos apuntes generales. México: Ecofronteras.

McBride, S. (1980). Un solo mundo, voces múltiples. UNESCO. París:

Fondo de Cultura Económica.

McQuail, D. y Van Cuilenburg, J. (2003). Cambios en el paradigma de política de medios. Hacia un nuevo paradigma de políticas de comunicación. European Journal of Communication, 18(2), 181-207.

Ortiz, R. (2004). Mundialización y Cultura. Colección Agenda Iberoamericana. Bogotá: Edición del convenio Andrés Bello.

Oslak, O. y O’Donnel, G. (1984). Estado y políticas estatales en América latina: hacia una estrategia de investigación. En Flores, G. (Comps.). Administración pública. Perspectivas críticas. Buenos Aires: ICAP

Postolski, G. (2003). Mucho ruido, pocas leyes. Economía y políticas de comunicación en la Argentina. Buenos Aires: La Crujía.

Postolski, G.; Santucho, A. y Rodríguez, D. (2001). Concentración y dependencia: Los medios de comunicación en el centro de la crisis. Buenos Aires: Observatorio Político y Social de Medios y del Área de Investigación de la UTP. 
Sierra, F. (2006). Políticas de comunicación y cultura. Barcelona: Gedisa.

Sinclair, J. (2000). Televisión: comunicación global y regionalización. Barcelona: Gedisa.

Williams, R. (1989). Resources of Hope: Culture, Democracy, Socialism. Londres: Verso. 